\title{
SAND\#2000-8607C
}

\section{Multi-Parameter High-Resolution Spatial Maps of a CdZnTe Radiation Detector Array}

\author{
N.R. Hilton ${ }^{1,2}$, H.B. Barber ${ }^{1}$, B.A. Brunett ${ }^{2.3}$, J.D. Eskin ${ }^{4}$, M.S. Goorsky ${ }^{5}$, R.B. James ${ }^{2}$, \\ J.C. Lund ${ }^{2}$, D.G. Marks ${ }^{1}$, T.E. Schlesinger ${ }^{3}$, T.M. Teska ${ }^{1}$, \\ J.M. Van Scyoc ${ }^{2}$, J.M. Woolfenden ${ }^{1}$, H. Yoon ${ }^{5}$ \\ 'University of Arizona, Tucson, Arizona 85724 \\ ${ }^{2}$ Sandia National Laboratories, Livermore, Califomia 94551 \\ ${ }^{3}$ Carnegie Mellon University, Pittsburgh, Pennsylvania 15213 \\ ${ }^{4}$ Panoptic Vision Inc., Boulder, Colorado 80303 \\ ${ }^{5}$ University of California, Los Angeles, Califormia 90095

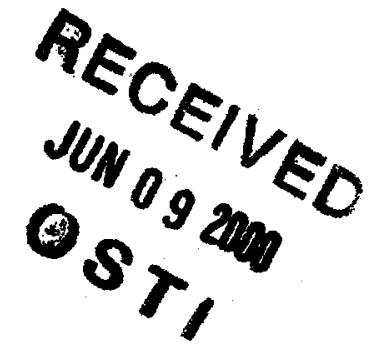

\section{Abstract}

Resistivity results from a $48 \times 48$ pixelated CdZnTe (CZT) radiation detector array are presented alongside X-ray topography and detector mapping with a collimated gammaray beam. By using a variety of measurements performed on the same sample and registering each data set relative to the others, the spatial dependence of relationships between them was examined. The local correlations between resistivity and one measure of detector performance were strongly influenced by the positions of grain boundaries and other gross crystal defects in the sample. These measurements highlight the need for material studies of spatially heterogeneous CZT to record position information along with the parameters under study.

\section{INTRODUCTION}

The purpose of this work is to utilize a $48 \times 48$ pixelated $\mathrm{Cd}_{0.8} \mathrm{Zn}_{02} \mathrm{Te}$ (CZT) radiation detector anray to measure detector properties at high spatial resolutions[1]. Detector array results are combined with data from other tests including $\mathrm{X}$-ray diffraction, photoluminescence, $\mathbf{I R}$ transmission imaging, and ${ }^{57} \mathrm{Co}$ gamma-ray mapping to provide a more comprehensive view of a single sample than has been available previously. This objective was prompted by some earlier work which sampled a few spots on a CZT crystal and suggested that increasing the number of samples would strengthen any conclusions relating to material parameters and performance as a radiation detector $[2]$.

This paper presents a subset of our results by focusing on the leakage current measurements made with the imaging array and gamma-ray mapping done after the pixels were removed. By measuring the physical and electrical properties of the CZT crystal at spatial resolutions comparable to the detector array spacing, we were able to examine the relationship between the local characteristics of the crystal and its performance. From a practical standpoint, this information should be considered when selecting material for manufacturing radiation detectors[3].

To avoid possible confusion caused by some terminology we define two terms which are used throughout this paper. "Resistivity" is the term we use to describe the results of the leakage current measurements. We are aware that this term may not always correspond to the physical resistivity of the
CZT substrate material (in the case of blocking contacts for instance). Nonetheless, we use the term resistivity because it is a convenient way of expressing the leakage current-scaled for pixel geometry and operating bias-that can be compared with the results reported by others. Second, we define "detector performance" to mean the total number of counts in the pulse height spectrum within an energy window (range of pulse heights). We realize that the counts in an energy window does not define all aspects of detector performance, but it is often a useful indicator of charge collection properties.

\section{EXPERIMENT}

The first tests performed with the imaging array were a series of leakage current measurements as a function of bias voltage and temperature. The device, shown in Figure 1 , is a $7 \times 7 \times 1.5 \mathrm{~mm}^{3} \mathrm{CZT}$ substrate on which a $48 \times 48$ array of gold pixels was delineated by photolithography at $125 \mu \mathrm{m}$ pitch. Visible on the opposite side is the continuous planar electrode. The detector array was indium bump bonded to a silicon multiplexer readout. This hybridized focal plane array was then wire bonded into a chip carrier. Several of these devices

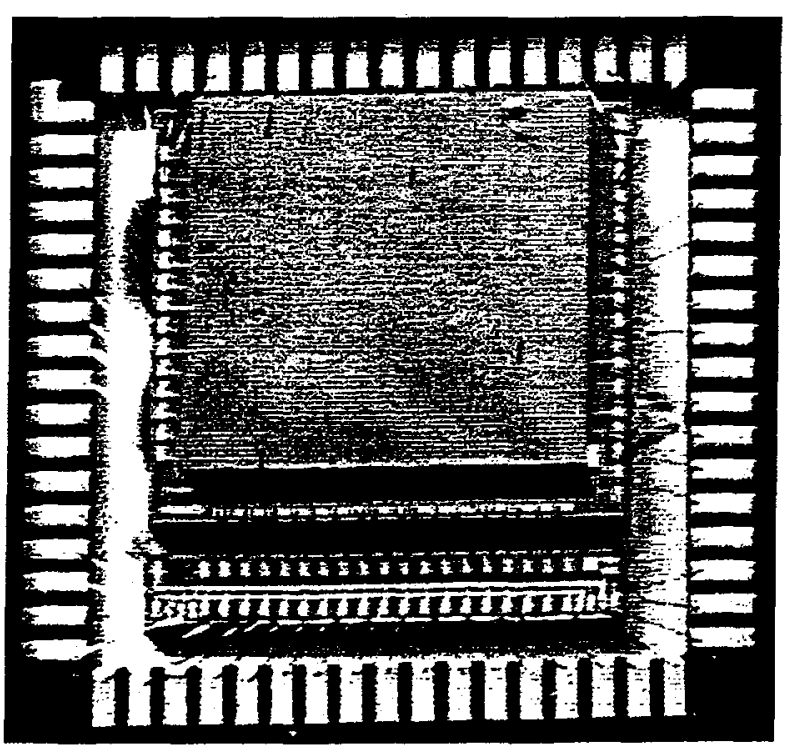

Figure 1: $48 \times 48$ focal plane array wire bonded in a chip carrier. This array was designed for high speed readout and low temperature operation. 


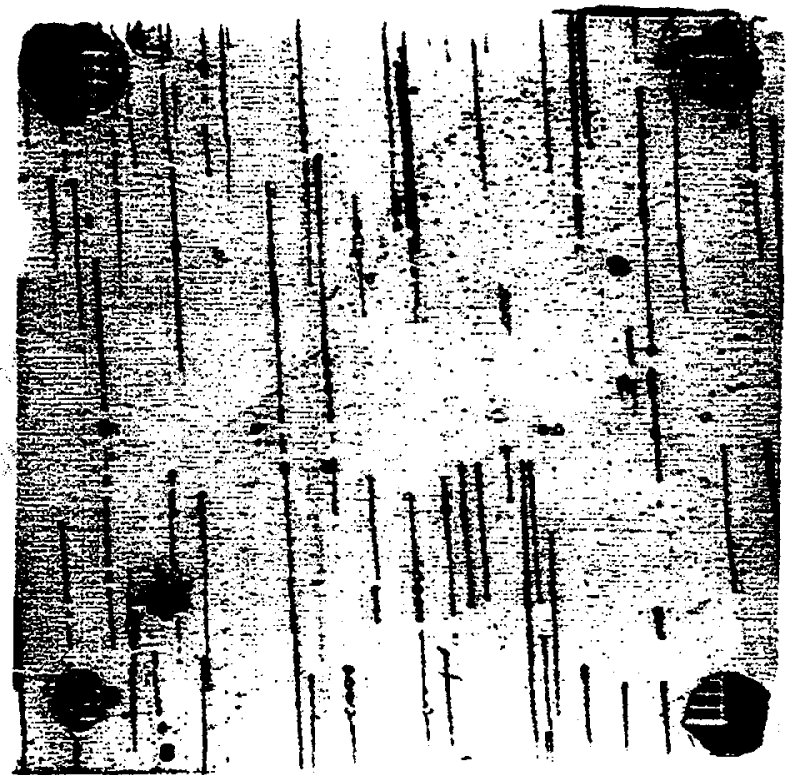

Figure 2: IR transmission photograph of the CZT crystal used in the $48 \times 48$ array. Pipes within crystal grains and small precipitates along boundaries are visible. Comparison with Figures 3 and 6 makes it easier to see some of these features. The five dark regions in the corners are remnants of the pixel array structure.

have been made and tested in a specially designed vacuum dewar that provides stable, controllable temperature of the devices as well as the front-end electronics. An external ADC, VME histogramming memory, and computer readout software convert the video signal from the array to histograms for each pixel[4].

The array readout technique integrates the charge induced on a pixel during each frame. Each unit cell of the multiplexer is sensitive to currents from thermally excited carriers as well as those from photon or particle interactions within a pixel's sensitive volume. At each voltage bias a leakage current measurement was obtained by noting the change in position of the "no-gamma" peak in the histogram for each pixel, relative to the zero bias offset. Currents were converted to resistivities assuming a fixed geometry for all pixels.

Later, the array was disassembled and most of the gold pixels were removed. A few were left as fiducial marks to register subsequent data sets. Although they are not reported here, these investigations included mapping with photoluminescence at room temperature and $4.2 \mathrm{~K}$, electron microprobe, and triple axis X-ray diffraction. We present data from infrared imaging, $X$-ray topography, and ${ }^{57} \mathrm{Co}$ gamma-ray mapping.

The IR transmission photograph in Figure 2 was taken with a $35 \mathrm{~mm}$ camera using a close-up lens and Kodak HEI film. $X$-ray topographs were taken at UCLA after determining crystal orientation from Laue diffraction patterns generated from locations on both the front and back faces of the sample. The "front" refers to the (formerly) pixelated side. Figure 3 is a topograph of the front side and the companion sketch in Figure 6 distills the major crystal grain information revealed when all the $\mathrm{X}$-ray and $\mathrm{IR}$ data are combined.

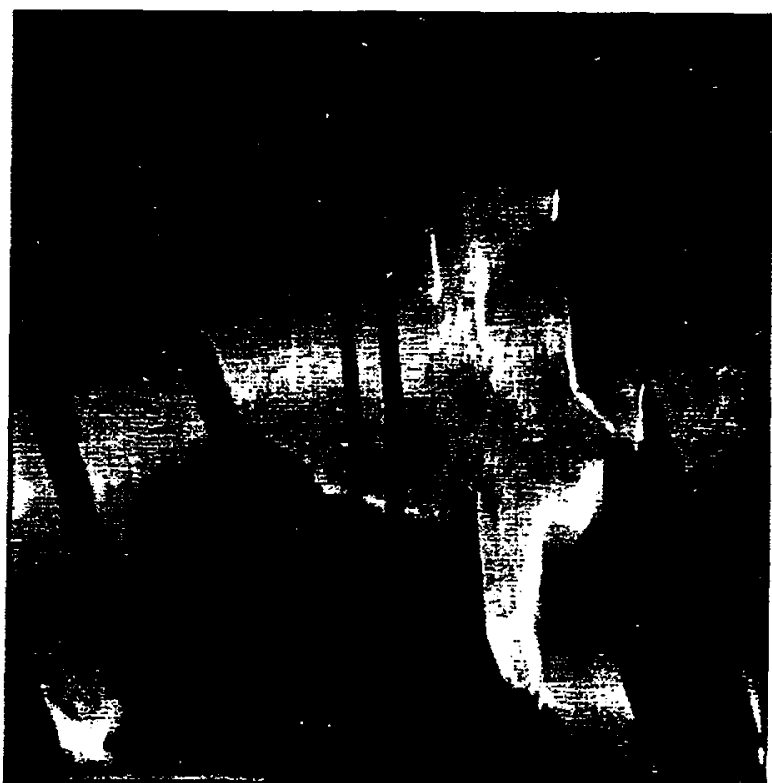

Figure 3: Double-crystal X-ray diffraction topograph (206 reflection). White areas are in the diffracting condition. In general, the dark areas in the lower portion of the image and extending into the middle are other crystal grains and twins. Except for twins, dark areas in the upper half are due to misorientation contrast since they are visible in other topographs.

The last test on this sample which we report here is the ${ }^{57} \mathrm{Co}$ gamma-ray mapping done at Sandia National Laboratory. Figure 4 shows the apparatus used. Single, planar, electroless gold contacts were deposited on both faces of the CZT crystal without removing the fiducial pixels. The detector was mounted inside a test box which rides on high-precision twoaxis translation stages and is connected to the charge sensitive pre-amplifier and high voltage supply. Pulse signals were digitized by an Ortec $A D C$ and histogramming memory package in a CAMAC crate. Gamma-ray spectra were recorded as a function of the position of collimator/source assembly over the detector.

The collimator is a stainless steel needle tube with a $200 \mu \mathrm{m}$ inside diameter embedded in a $1 \mathrm{~cm}$ thick eutectic alloy of $44.5 \%$ lead and $55.5 \%$ bismuth. This alloy melts at $255^{\circ} \mathrm{F}$ which makes fabrication easier and safer than if it were

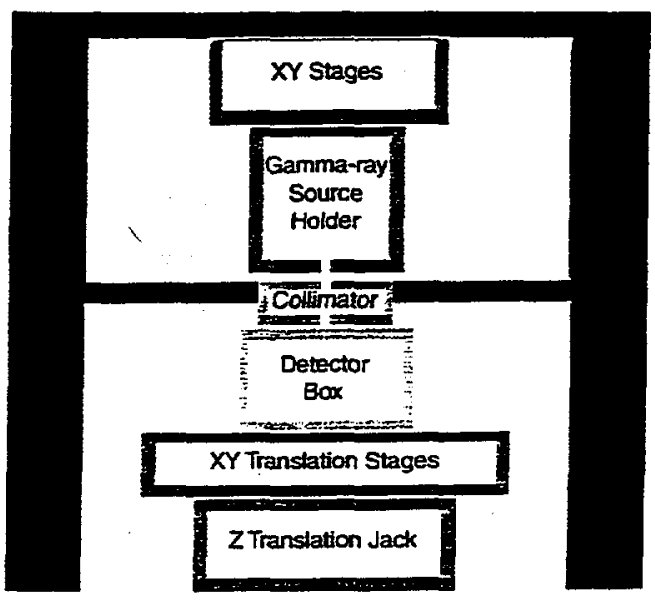

Figure 4: Sandia Labs ${ }^{57}$ Co gamma-ray mapping apparatus. 


\section{DISCLAIMER}

This report was prepared as an account of work sponsored by an agency of the United States Government. Neither the United States Government nor any agency thereof, nor any of their employees, make any warranty, express or implied, or assumes any legal liability or responsibility for the accuracy, completeness, or usefulness of any information, apparatus, product, or process disclosed, or represents that its use would not infringe privately owned rights. Reference herein to any specific commercial product, process, or service by trade name, trademark, manufacturer, or otherwise does not necessarily constitute or imply its endorsement, recommendation, or favoring by the United States Government or any agency thereof. The views and opinions of authors expressed herein do not necessarily state or reflect those of the United States Government or any agency thereof. 


\section{DISCLAIMER}

Portions of this document may be illegible in electronic image products. Images are produced from the best available original document. 
pure lead. After cooling, the protruding tube was machined off leaving the inside of the tube relatively free of debris. A quick acid rinse cleared any remaining material inside the collimator bore. A custom ${ }^{77} \mathrm{Co}$ source with a $1 \mathrm{~mm}$ diameter active area was placed in a shielded source holder made of the same alloy and positioned over the collimator. Because a high activity "Co source was needed to get a sufficient count rate at $122 \mathrm{keV}$, the 570 and $692 \mathrm{keV}$ gamma rays-for which shielding is relatively ineffective-contribute a significant number of counts in the pulse height spectra. Data acquisition is computer controlled by software written with LabVIEW. Further discussion and results involving this gamma-ray mapping apparatus are reported elsewhere in these proceedings[5].

\section{RESULTS AND DISCUSSION}

The results of the leakage current tests are presented in Figure 5. The histogram data were transformed into leakage currents and then into resistivities. Figure 5 is a rescaled map of these resistivity values where extremely high and low values have been clipped to allow more subtle features to be visible. Lighter areas indicate higher resistivities. Black areas are where no resistivity values were measured. Several hundred of those pixels for which we have no data had indium bumps that were delaminated from the multiplexer circuit. Typically, the delaminated pixels were on the periphery of the array. We camnot report resistivity values for many other pixels (a few hundred) which were mostly located between the delaminated pixels and the good pixels because they had leakage currents beyond the dynamic range of the readout electronics.

A depiction of the crystallographic information on this sample is shown in Figure 6. The two crystal grains studied by

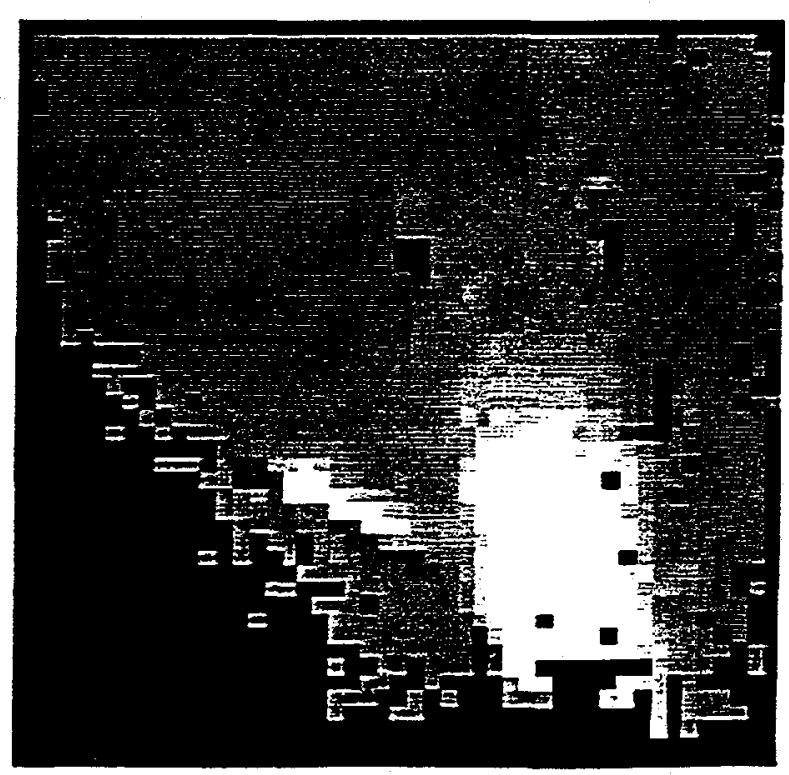

Figure 5: Map of pixel resistivity at $30^{\circ} \mathrm{C}$. Solid black areas are where no reliable resistivity data was found. Lighter regions indicate higher resistivity. Resistivity values in white areas have been clipped to increase grayscale contrast of more subtle features.
$X$-ray topography on the fromt and back sides of the crystal are not the same grains. Referring to Figure 6, the diffraction condition was met on the front side in regions 1 and 5 and on the back in regions 4,5 , and 6 . One notable missing piece of information is what direction the twins (clearly visible in Figure 3 as dark bands) extend into, and then possibly exit the crystal on the back side. Furthermore, we must allow that regions in both topographs which were not in the diffraction condition could be more than one grain themselves. Thus, in Figure 6 we have indicated with a question mark where an additional grain boundary may intersect the front surface which would mean the grain(s) of region 6 is different from the grain(s) of 3 and 4 . We suggest that the grains are different and that regions 3 and 4 comprise a single grain which extends up and to the right as it enters the crystal. This grain then exits in regions 4,5 , and 6 on the back.

If the proposed configuration is correct, the perimeters of these sets of regions are connected by a grain boundary. The dotted line in Figure 6 indicates the extent of the boundary on the interior of the crystal. This boundary is visible in Figure 2 due to precipitates which often decorate such boundaries. A second grain enters in region 6 and exits out the side resulting in at least one additional boundary traversing the sample in this area. Many other grain configuration possibilities exist for this sample which cannot all be represented or investigated in this paper. We present one possible configuration and the supporting evidence. The square box in Figure 6 indicates the location of the pixel array and the only region imaged in Figures 5,8 , and 12. It will often be useful to refer to the boxed region in Figure 6 in the remainder of the paper.

Gamma-ray detection performance data from this array have been reported earlier along with various methods to improve the spatial and spectral resolution[6-8]. The pixels

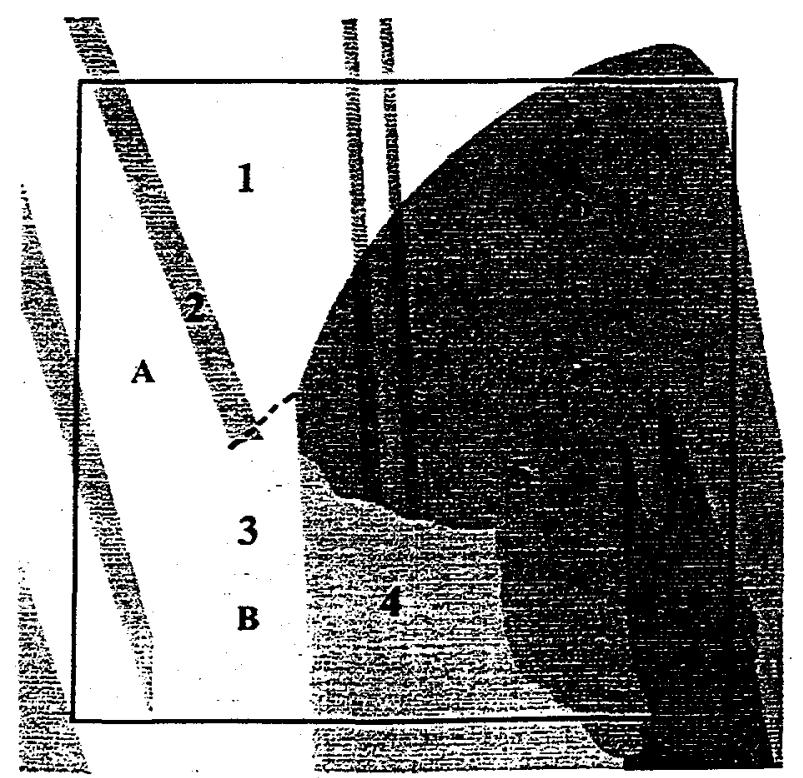

Figure 6: A representation, derived from the IR photo and $X$-ray topographs, of where known crystal grains intersect the front and back surfaces. The black square marks the location of the pixel array. See text for descriptions of other markings. 
are sensitive mainly to one type of charge carrier because of their small size $[4,9,10]$. Hole tailing of the spectra caused by dissimilar charge transport properties for holes and electrons when photons interact throughout the detector is therefore reduced. However, because of non-localized charge production and transport, too much charge is spread to neighboring pixels to consistently generate the same induced charge on a pixel for photon interaction events within its volume. Consequently, the photopeak is degraded unless processing of the data is done to compensate. Nevertheless, we show here that pixel geometry is not the only factor which determines detector performance.

Unfortunately, limited gamma-ray performance data on the CZT sample were obtained while operated as an imaging array because it was damaged during testing. Instead, the sample was re-fabricated into a planar device by applying a continuous gold electrode on each side of the sample. Gamma-ray pulse height spectra as a function of position on the device were then measured using the mapping apparatus described earlier. Figures 7-9 show some results from these collimated ${ }^{57} \mathrm{Co}$ gamma-ray mapping studies. Two typical pulse height spectra acquired at locations marked $A$ and $B$ in Figure 6 are shown in Figure 7. The region between the vertical dotted lines is the integration window used to generate Figure 8, which is a grayscale map of the total counts recorded within this energy window at each point on the sample.

The feature that is immediately evident when interpreting Figures 7 and 8 is that crystal grain boundaries shift the photopeaks of the spectra to lower energies. The grain boundaries impede charge transport from one grain to the next. This results in less than maximum charge induction on the electrodes[11]. Since the gamma rays interact in the crystal on both sides of the grain boundary, we would expect to see duplicate photopeaks in the pulse height spectrum at the energy difference between the new energies of the original photopeaks and their energies measured at a location without a grain boundary. The 122 and $136 \mathrm{keV}$ peaks unite and become one as they shift so there are only two peaks in the spectra - the original and the duplicate of the united photopeak. For an example, compare spectrum " $A$ " in Figure 7 with spectrum " $a$ " in Figure 9. Furthermore, with our mapping data we can infer the depth and slope of the grain boundary from the ratios between the energies of the original and duplicate photopeaks. This can be seen in the spectra shown in Figure 9 when referenced to their respective sampling locations in Figure 6. These data support our hypothesis about the position of the main grain boundary described earlier.

We also investigated if there were correlations between resistivity, crystal topography, and detector performance. Figure 10 shows a scatter plot comparing pixel resistivity and integrated counts within the energy window. This measure of detector performance is a reasonable representation of the charge transport efficiency at a given location on the detector. Correlations within scatter plot data such as this would be indicated by diagonal patterns; conversely, vertical and

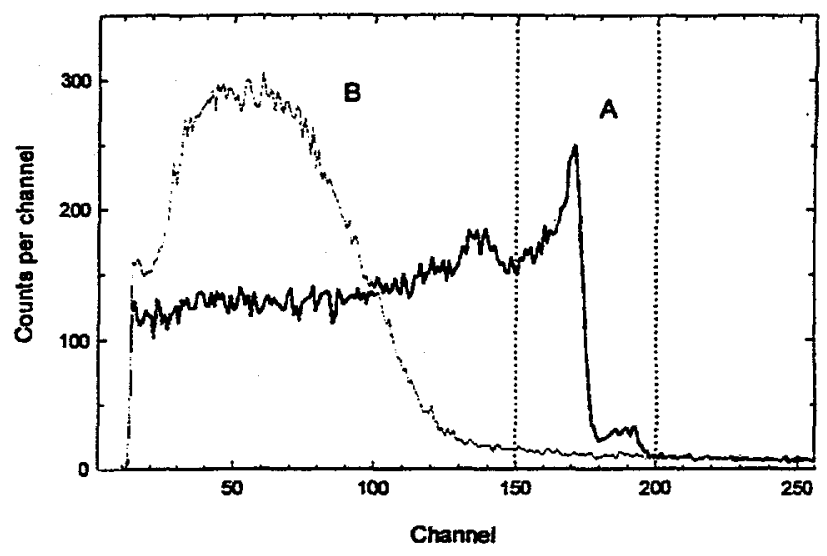

Figure 7: Pulse height spectra of a collimated ${ }^{57}$ Co source irradiating locations $A$ and $B$ shown in Figure 6. The dotted lines indicate the energy window used to produce the image in Figure 8.

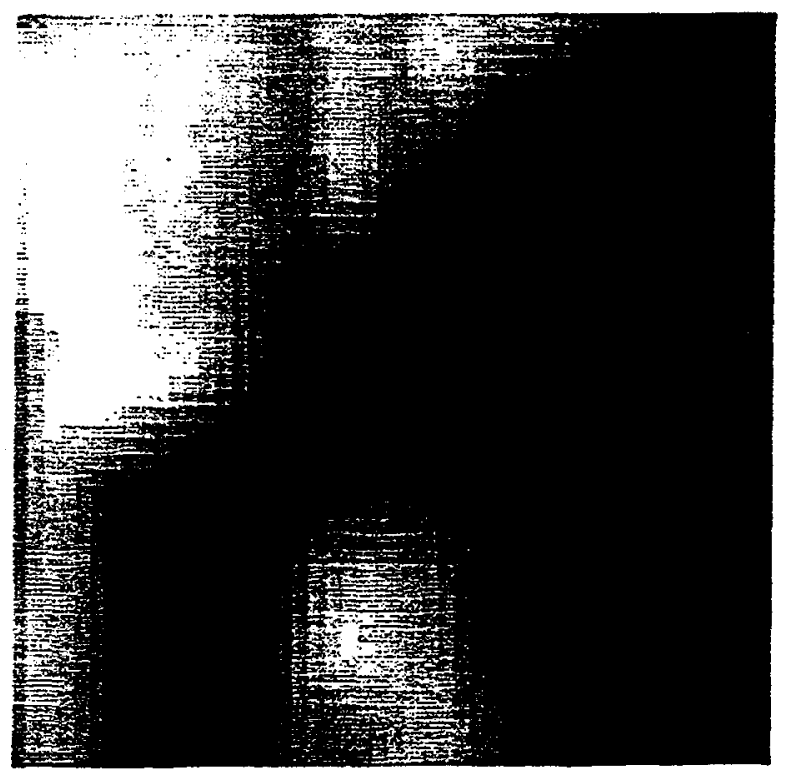

Figure 8: Map of integrated counts within the energy window shown in Figure 7 as a function of position on the sample. The effect of both high angle grain boundaries and twins is obvious.

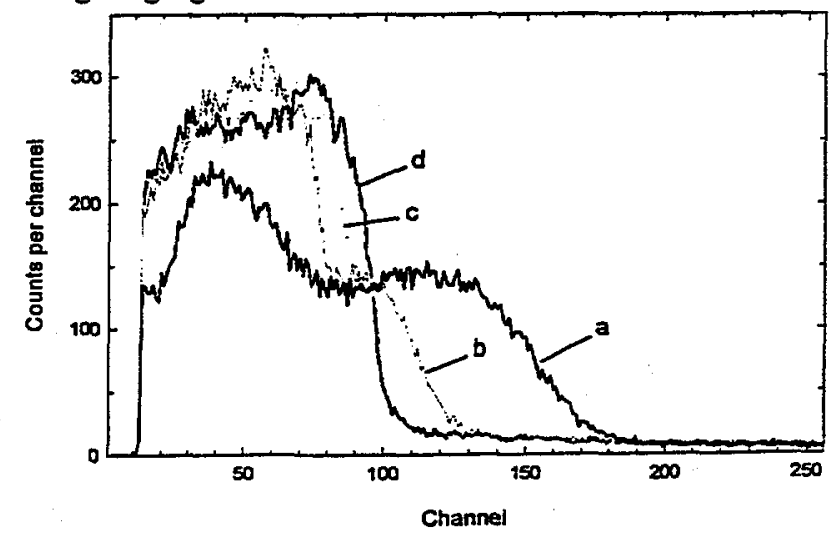

Figure 9: Pulse height spectra obtain at locations a, b, c, and d shown in Figure 6 . This region contains a crystal grain boundary. The detector effectively behaves as two detectors whose relative thickness are proportional to their relative photopeak positions. The resulting spectrum is the superposition of the spectra from within each grain. 


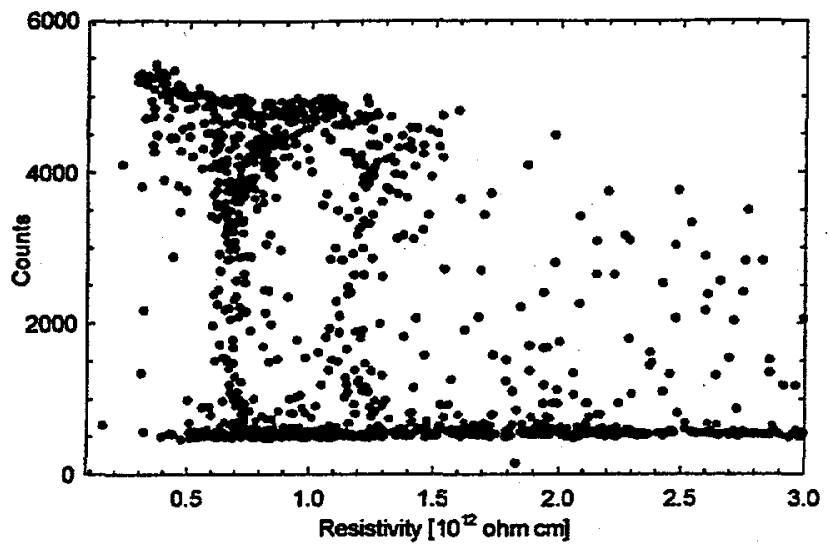

Figure 10: A scatter plot of integrated counts in the high energy window versus resistivity at $30^{\circ} \mathrm{C}$ suggests there may be small correlations, possibly in localized regions.

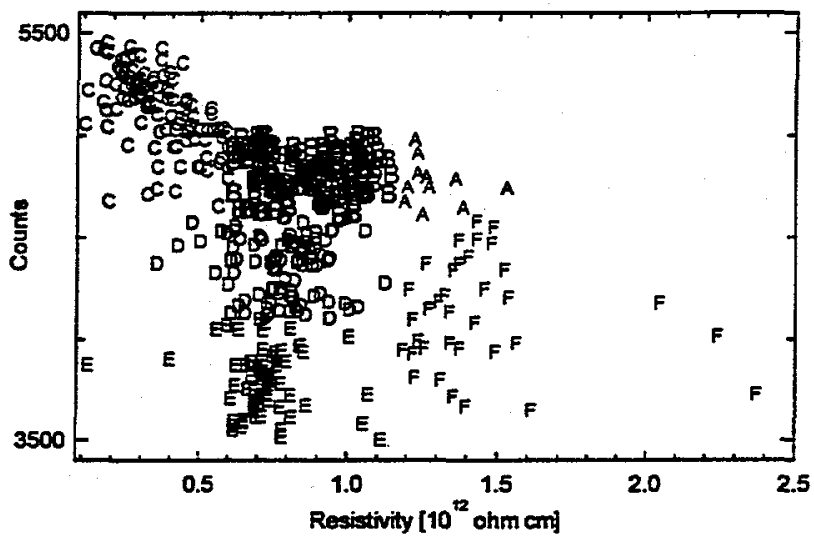

Figure 11: Eniarged version of previous plot where only pixels in the upper left comer are shown, clustered into groups according to their location on this scatter plot See Figure 12 for a map showing how each lettered group is spatially distributed.

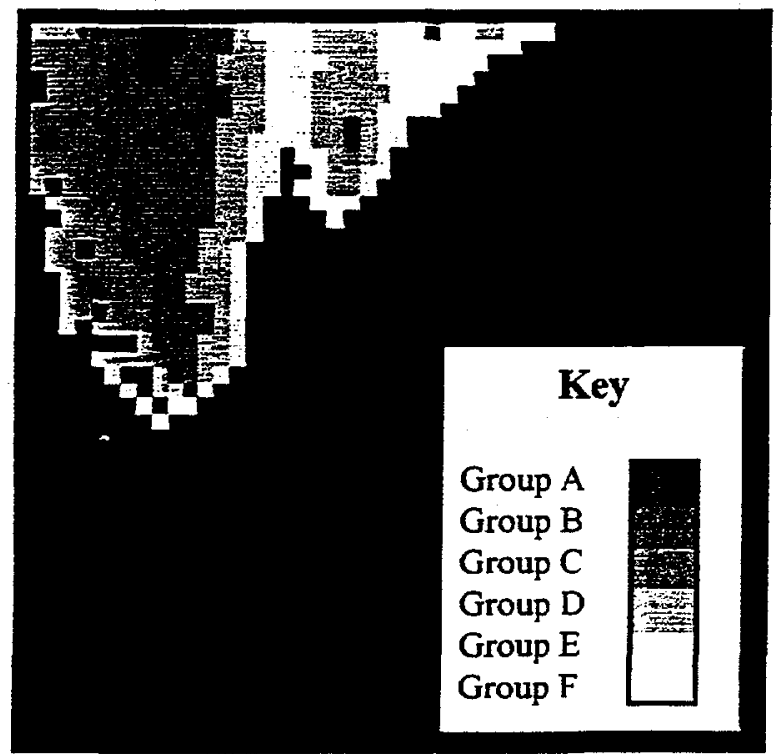

Figure 12: Locations of pixels plotted in Figure 11 according to their classifications by resistivity and detector performance. Black regions either do not have resistivity data or are not considered here because of location or the number of counts in energy window. horizontal patterns describe uncorrelated parameters. Clearly, Figure 10 shows no obvious correlation between charge transport efficiency and resistivity. The lack of correlation is somewhat surprising since impurities in the bulk semiconductor might be expected to affect both resistivity and charge transport. It is possible that the presence of grain boundaries so dominates the charge transport that any possible correlations are overshadowed.

In order to study the relationship of resistivity to detector performance without the effects of grain boundaries we looked only at pixels in the upper left-hand comer of the device. In spite of the twins in this area, we concluded that it was the only region which could contain areas free of grain boundaries. Figure 11 is an enlarged version of the scatter plot for this region with the data grouped into several classes. The spatial distribution of these classes is shown in Figure 12.

Although there is some evidence for localized structure in groups $\mathrm{C}$ and $\mathrm{B}$ relating resistivity and performance, there are still no convincing indications that these quantities are correlated. Given that the measured resistivity varied by over an order of magnitude, the absence of a strong correlation indicates that we must look elsewhere for a mechanism to account for the detector performance variation. The dominant structure in Figure 11 is likely related to the twins in this part of the detector, as seen in Figure 12.

\section{CONCLUSIONS}

The original motivation for this work was the possibility of gathering data at a large number of points on a CZT sample to determine what kinds of relationships exist between measured material parameters and the performance of radiation detectors constructed from this material. In particular, we wished to assess the influence of physical and electrical characteristics of a CZT crystal on the efficiency and energy resolution of a small pixel detector array made from that crystal. In this paper we focused on correlating a subset of our physical properties data (resistivity and crystal grain locations) with the pulse height spectra obtained from gamma-ray excitation. Because we knew the physical location from which each physical property datum was collected, and we were able to acquire a gamma-ray pulse height spectrum with similar position resolution, we could interpret the local correlations visible in this particular sample.

The measurements described in this paper were conducted on just one sample. However, this particular sample was so heterogeneous and the amount of data was so large that we can infer some general conclusions about the effects of CZT crystal properties on radiation detector performance. The most obvious conclusion is that grain boundaries, visible in $\mathbb{R}$ transmission microscopy and X-ray topography, have an extremely deleterious effect on the radiation detector characteristics. The correlation of such a gross physical defect as a grain boundary with detector pixel performance is hardly surprising and has been noted by others[11]. Nonetheless, this seems to be the first instance that quantitative evidence linking 
poor radiation detector performance with adjacent grain boundaries is presented.

A surprising conclusion of this work is that there was no evidence of a correlation between resistivity and our measure of performance, or charge transport efficiency. The dominant effects of the grain boundaries are so striking that the intragrain response noted in this paper seems subtle in comparison. It seems clear that resistivity maps are poor indicators for selecting good crystals for detectors using a pulse electronics readout. Of course, such maps are useful in cases where integrating readout methods such as our multiplexer approach are used, since too much leakage current offset can drive individual pixels out of the dynamic range of the electronics.

Clearly the work presented here supports the conclusion that grain boundaries have a very large negative effect on the characteristics of radiation detectors, and single crystals of CZT should be used in manufacturing detectors whenever possible. We look forward to the opportunity to view detectors manufactured from more homogeneous crystals using the methods developed for this study. Such future investigations should enable us to obtain a better quantitative understanding of the relationship between material parameters such as resistivity and charge collection properties.

\section{ACKNOWLEDGMENTS}

The author gratefully acknowledges the support of his coauthors for the facilities and persomel made available for this work. Funding for the focal plane array was received through NIH grant RO1 CA 75288. Sandia National Laboratories are operated under contract number DE-AC04-94AL85000 for the DOE by Sandia Corporation, a Lockheed Martin Company. Specifically, Laboratory Directed Research and Development and the Office of Nuclear Nomproliferation supported the Sandia portion of this work.

\section{REFERENCES}

[1] H.B. Barber, et al., "Use of CdZnTe pixel arrays with multiplexer readout to map detector crystal properties," 1997 IEEE NSS/MIC Conference Record.

[2] J.E. Toney, et al., "Uniformity of $\mathrm{Cd}_{1-x} \mathrm{Zn}_{\mathrm{x}} \mathrm{Te}$ grown by high-pressure Bridgman," Nuclear Instruments and Methods in Physics Research A, vol. 380, pp.132-135, 1996.

[3] J.C. Lund, et al., "Semiconductor material requirements of orthogonal strip detectors," SPIE Proceedings, vol. 3115 , pp.190-198, 1997.

[4] J.D. Eskin dissertation, Semiconductor Gamma-ray Detectors for Nuclear Medicine, University of Arizona, 1997.

[5] B.A Brunett, et al., "Fine-scale spatial response of CdZnTe radiation detectors," 1998 IEEE NSS/MC Conference Record.

[6] H.B. Barber, et al, "Semiconductor pixel detectors for gamma-ray imaging in nuclear medicine," Nuclear Instruments and Methods in Physics Research $A$, vol. 395, pp.421-428, 1997.

[7] D.G. Marks, et al., "A $48 \times 48$ CdZnTe array with multiplexer readout," IEEE Transactions on Nuclear Science, vol. 43, no. 3, pp.1253-1259, 1996.

[8] D.G. Marks, et al., "Maximum-likelihood estimation for semiconductor detector arrays," 1997 IEEE NSS/MIC Conference Record.

[9] D.J. Wagenaar and R.A. Terwilliger, "Effects of induced charge in the kinestatic charge detector," Medical Physics, vol. 22, no. 5, pp.627-634, May 1995.

[10] J.D. Eskin, et al., "The effect of pixel geometry on spatial and spectral resolution in a CdZnTe imaging array," 1995 IEEE NSS/MIC Conference Record.

[11]F.P. Doty, J.P. Cozzatti, and J.P. Schomer, "Characterization of bulk CdZnTe by IR transmission imaging," SPIE Proceedings, vol. 3115, pp.51-55, 1997. 